\title{
ON TRUNCATIONS OF HANKEL AND TOEPLITZ OPERATORS
}

\author{
Aline Bonami And Joaquim Bruna
}

Abstract

We study the boundedness properties of truncation operators acting on bounded Hankel (or Toeplitz) infinite matrices. A relation with the Lacey-Thiele theorem on the bilinear Hilbert transform is established. We also study the behaviour of the truncation operators when restricted to Hankel matrices in the Schatten classes.

\section{Statement of results}

In this note we will be dealing with infinite matrices $B=\left(b_{m, n}\right)_{m, n \geq 0}$, $b_{m, n} \in \mathbb{C}$, which we identify with linear operators on $l^{2}(\mathbb{N})$. More precisely we assume that

$$
\sup _{n} \sum_{m}\left|b_{m, n}\right|^{2}<\infty
$$

and consider $B$ as defined on almost finite sequences. Also we identify $l^{2}(\mathbb{N})$ with the Hardy space $H^{2}(\mathbb{D})$ of the unit disc and consider $B$ as a linear map from the space of polynomials to $H^{2}(\mathbb{D})$. One property we will be considering is whether $B$ extends to a bounded operator on the whole space, that is

$$
\sum_{m}\left|\sum_{n} a_{n} b_{m, n}\right|^{2} \leq\|B\|^{2} \sum_{n}\left|a_{n}\right|^{2} .
$$

The matrix $B$ is called of Toeplitz type if $b_{m, n}=b_{m-n}$ for some sequence $b \in l^{2}(\mathbb{Z})$, and we write $B=T_{b}$. We identify $b$ with the $L^{2}$ function $b(\zeta)=\sum_{j=-\infty}^{+\infty} b_{j} \zeta^{j}$ on $\mathbb{T}=\partial \mathbb{D}$, called the symbol. In this case it is immediate to see that $T_{b}$ can be realized as the integral operator from $H^{2}(\mathbb{D})$ to the space of holomorphic functions on $\mathbb{D}$ given by 
$T_{b} f=C(b f)$, where $C$ is the Cauchy integral giving the projection from $L^{2}(\mathbb{T})$ to $H^{2}(\mathbb{D})$ : if $f=\sum_{n} a_{n} z^{n}$,

$$
T_{b}(f)(z)=\frac{1}{2 \pi} \int_{\mathbb{T}} \frac{b(\zeta) f(\zeta)}{1-\bar{\zeta} z} d \sigma(\zeta)=\sum_{m \geq 0}\left(\sum_{n \geq 0} a_{n} b_{m-n}\right) z^{m}, z \in \mathbb{D} .
$$

It is well known that $T_{b}$ is a bounded operator on $H^{2}(\mathbb{D})$ if and only if $b \in L^{\infty}(\mathbb{T})$, with the same norms.

The matrix $B$ is called of Hankel type if $b_{m, n}=b_{m+n}$ for some sequence $b \in l^{2}(\mathbb{N})$, and we write $B=H_{b}$. In this case the symbol is the $H^{2}$ function $b(\zeta)=\sum_{j=0}^{+\infty} b_{j} \zeta^{j}$ and $H_{b}$ can be realized as the integral operator $H_{b} f=C(b g)$, where $g$ denotes the antiholomorphic function $g(\zeta)=f(\bar{\zeta})$,

$$
H_{b}(f)(z)=\frac{1}{2 \pi} \int_{\mathbb{T}} \frac{b(\zeta) f(\bar{\zeta})}{1-\bar{\zeta} z} d \sigma(\zeta)=\sum_{m \geq 0}\left(\sum_{n \geq 0} a_{n} b_{m+n}\right) z^{m}, z \in \mathbb{D}
$$

It is well known that $H_{b}$ is a bounded operator on $H^{2}(\mathbb{D})$ if and only if $b \in B M O A(\mathbb{T})$, again with comparable norms, and compact if and only if $b \in V M O A(\mathbb{T})$. In these cases, $b$ is the holomorphic part of some bounded (resp. continuous) function and since the antiholomorphic part gives zero contribution in the above integral, one can assume that $H_{b}$ is given by the above integral with $b \in L^{\infty}(\mathbb{T})($ resp. $b \in C(\mathbb{T})$ ).

We consider truncations of matrices defined as follows. For real $\beta, \gamma$ and a matrix $B$ as before, we let $\Pi_{\beta, \gamma}(B)$ the matrix whose entry at position $m, n$ is $b_{m, n}$ when $m \geq \beta n+\gamma$ and zero otherwise. For $\beta=1$, $\gamma=0$ we use the notation $\Pi$. For $\beta=0, \Pi_{0, \gamma}(B)$ is just $B$ followed by a projection, and hence we will only consider $\beta \neq 0$.

For general $B$, boundedness of $B$ does not imply boundedness of $\Pi_{\beta, \gamma}(B)$, for $\beta>0$. For instance, if $B=T_{b}$ with $b$ bounded then $\Pi(B)$ is also a Toeplitz operator whose symbol is $C b$, the holomorphic projection of $b$. Since $C b$ is not necessarily bounded, $\Pi(B)$ may be an unbounded operator. For $\beta<0, B-\Pi_{\beta, \gamma}(B)$ has finite rank and hence $\Pi_{\beta, \gamma}(B)$ is always a bounded operator. In this case the natural question is whether the operator norms $\left\|\Pi_{\beta, \gamma}(B)\right\|$ are bounded independently of $\gamma$. This is again false, in this case a Hankel operator gives a counterexample. Indeed, if $B=H_{b}$ with $b \in B M O A(\mathbb{T})$, then $\Pi_{-1, N}(B)$ are again Hankel operators with symbols $S_{N} b$. Here $S_{N} b(\zeta)=\sum_{j=0}^{N} b_{j} \zeta^{j}$, and a counterexample is given by $b \in B M O A$ such that the $S_{N} b$ are not uniformly bounded in $B M O$. 
Within special classes of operators, the truncations $\Pi_{\beta, \gamma}$ have a good behaviour. For instance, it is obvious that they preserve the HilbertSchmidt class $S_{2}$, defined by the condition $\sum_{m, n}\left|b_{m, n}\right|^{2}<\infty$, and more generally it can be shown that they preserve the Schatten classes $S_{p}$ for $p>1$; a proof is included in the last section. It is a well known fact for triangular truncations, as one can see in [DS] or [GK2]. For $p=1$ and the trace class $S_{1}$ this is no longer true (this will be shown in the last section too). It is customary to present the above situation as analogous to the behaviour of the Hilbert transform or the Cauchy projection $C$ in the range of $L^{p}$-spaces: $\Pi$ plays the role of Hilbert transform on matrices and the classes $S_{p}$ are, by definition $([\mathbf{G K 1}],[\mathbf{P o}]), L^{p}$ classes of operators.

Our first result establishes that with the exception of the counterexamples above, the truncations $\Pi_{\beta, \gamma}$ behave well when restricted to bounded Hankel or Toeplitz operators:

Theorem 1. (a) If $B$ is a bounded (resp. compact) Hankel operator, and $\beta \neq-1, \Pi_{\beta, \gamma}(B)$ are bounded (resp. compact) operators. Moreover their norms are uniformly bounded in $\gamma$, and locally uniformly bounded in $\beta$ away from 0 and -1 .

(b) If $B$ is a bounded Toeplitz operator, and $\beta \neq 1, \Pi_{\beta, \gamma}(B)$ are bounded operators. Moreover their norms are uniformly bounded in $\gamma$, and locally uniformly bounded in $\beta$ away from 0 and 1.

Our proof consists in exhibiting an integral operator realizing $\Pi_{\beta, \gamma}(B)$, closely related to the bilinear Hilbert transform, and applying the a priori estimates of the Lacey-Thiele theorem $([\mathbf{L T 1}],[\mathbf{L T 2}],[\mathbf{L T 3}])$. We do not know of any "operator theory proof" of the result.

In the last section we consider Hankel operators in the trace class $S_{1}$. A theorem of Peller $([\mathbf{P e}])$ describes the corresponding symbols as those in the Besov space $B^{1}$. In contrast with the result above, we show that, for such $B, \Pi(B)$ is not in general in the trace class, and we give a sufficient condition on the symbol for this to be the case.

Let us mention that the truncation operators have specially been under consideration when $\beta= \pm 1$. See $[\mathbf{K P}]$ for considerations on the norms of $\Pi_{-1, \gamma}$, and $[\mathbf{A C N}]$ for precise estimates of the norm of $\Pi$. Let us also mention that the theorem gives a positive answer to a conjecture attributed to V. Peller.

\section{Proof of the theorem}

We consider first the case of Hankel operators. 
Assume first that $B=H_{b}$ is bounded, and let $b \in L^{\infty}(\mathbb{T})$ as in (1), with holomorphic part $\sum_{j \geq 0} b_{j} \zeta^{j}$. For $\beta \neq-1$ we must see that, independently of $\gamma$,

$$
\sum_{m \geq 0}\left|\sum_{m \geq n \beta+\gamma} b_{m+n} a_{n}\right|^{2} \leq C(\beta)\|b\|_{\infty}^{2} \sum_{n \geq 0}\left|a_{n}\right|^{2}
$$

It is enough to prove that this holds for rational $\beta, \gamma$ with a constant $C(\beta)$ which is locally bounded away from -1 and 0 . Without loss of generality we may assume that $\beta=\frac{k}{l}$ with $k, l \in \mathbb{Z}, k \geq 1, k \neq-l$, and $l \gamma \in \mathbb{Z}$. Moreover, we may assume that both $b, f$ are trigonometric polynomials; this is clear for $f$, and for $b$ it follows from the existence of a sequence $\left(b_{N}\right)$ of trigonometric polynomials, uniformly bounded by $\|b\|_{\infty}$, whose Fourier coefficients $c_{k}\left(b_{N}\right)$ equal $c_{k}(b)$ for $|k| \leq N$ (for instance using the de la Vallée-Poussin kernel).

Now, for $b$ a trigonometric polynomial, we consider an operator $A_{b}$ acting on periodic functions as follows. For $f \in L^{2}(\mathbb{T})$,

$$
\begin{aligned}
A_{b} f(x) & =\text { p.v. } \frac{1}{2 \pi} \int_{-\pi}^{\pi} b(k x+l t) f(t) e^{-i t l \gamma} \frac{d t}{\tan \frac{x-t}{2}} \\
& =\text { p.v. } \frac{1}{2 \pi} \int_{-\pi}^{\pi} b((k+l) x-l t) f(x-t) e^{-i \gamma l(x-t)} \frac{d t}{\tan \frac{t}{2}}
\end{aligned}
$$

We will make use of the following result by Lacey-Thiele in [LT3]:

Theorem 2. Let $\alpha \in \mathbb{R}, \alpha \neq 0, \neq-1$, and let $1<p, q \leq+\infty$ with $\frac{1}{r}=\frac{1}{p}+\frac{1}{q}<\frac{3}{2}$. Then there exists a constant $C(\alpha, p, q)$ such that for $f, g$ in the Schwartz class $\mathcal{S}(\mathbb{R})$ the bilinear Hilbert transform

$$
H_{\alpha}(g, f)(x)=p . v . \int_{-\infty}^{+\infty} g(x+\alpha t) f(x-t) \frac{d t}{t}
$$

satisfies the inequality

$$
\|H(g, f)\|_{r} \leq C(\alpha, p, q)\|f\|_{p}\|g\|_{q}
$$

Moreover the constant $C(\alpha, p, q)$ is locally bounded as a function of $\alpha$. 
If $C(\alpha, p, q)$ denotes the best constant satisfying the above inequality, note that $C(\alpha, p, q)=C\left(\alpha^{-1}, q, p\right)$. Also note that in the limiting cases, by the boundedness properties of the Hilbert transform, $C(0, p, q)$ is finite if $1<p<\infty, 1 \leq q \leq+\infty$, and $C(-1, p, q)$ is finite if $1<r<\infty$.

Let us transport the Lacey-Thiele result to the periodic situation. For a couple of integers $k, l, k \neq l$, we define the bilinear conjugate transform by

$$
H_{k, l}(g, f)(x)=\text { p.v. } \frac{1}{2 \pi} \int_{-\pi}^{\pi} g(k x+l t) f(t) \frac{d t}{\tan \frac{x-t}{2}},
$$

with $f, g \in C^{\infty}(\mathbb{T})$. Note that $H_{k, l}(g, f)$ is again periodic. We claim that there exists a constant $C(k, l, p, q)$, with the same conditions on $p, q$, such that

$$
\left\|H_{k, l}(g, f)\right\|_{r} \leq C(k, l, p, q)\|f\|_{p}\|g\|_{q} .
$$

To show (3), we first observe that, writing the periodic function $f$ as a sum of three functions which are periodizations of functions whose support is contained in an interval of length $\pi$ and using translation invariance, we may assume that $f$ is supported in $[-\pi / 2, \pi / 2]$. Consider now $H_{k, l}(g, f)(x)$ for $|x| \leq \pi$. Then $|x-t| \leq \frac{3 \pi}{2}$, and $\left(2 \tan \frac{x-t}{2}\right)^{-1}$ can be written $(x-t)^{-1}+a(x-t)$ where $a$ is a smooth function. The term with $a$ gives in $H_{k, l}$ a term which is pointwise bounded by

$$
\int_{-\pi / 2}^{\pi / 2}|g(k x+l t)||f(t)| d t
$$

and which, by the continuous Minkowski inequality, has finite $L^{q}$ norm bounded by $\|f\|_{1}\|g\|_{q}$. Since $r \leq q$, this term satisfies the required estimate in (3). It remains to consider

$$
\text { p.v. } \int_{-\pi / 2}^{\pi / 2} g(k x+l t) f(t) \frac{1}{x-t} d t
$$

For $|x| \leq \pi$, this equals

$$
\text { p.v. } \int_{-\infty}^{+\infty} G(k x+l t) F(t) \frac{1}{x-t} d t
$$


where $F(t)=f(t)$ in $|t| \leq \pi / 2$ and 0 elsewhere, and $G=g \phi, \phi$ being some cutoff function which is 1 for $|\xi| \leq|k| \pi+|l| \frac{\pi}{2}$. But this last expression is just $H_{\alpha}(\tilde{G}, F)(x)$, with $\alpha=-\frac{l}{k+l}$ and $\tilde{G}(\xi)=G((k+l) \xi)$, and hence (3) follows with

$$
C(k, l, p, q) \leq C_{0}+C_{0}|k+l|^{-\frac{1}{q}} C\left(-\frac{l}{l+k}, p, q\right)
$$

for some constant $C_{0}$. Applying (3) with $g=b, q=\infty, p=r=2$, we thus obtain

$$
\left\|A_{b} f\right\|_{2} \leq C(\beta)\|b\|_{\infty}\|f\|_{2}
$$

for $f, g$ trigonometric polynomials. Let us now check how $A_{b}$ works in the usual basis of exponentials. It is enough to consider $b(t)=e^{i j t}$ and $f(t)=e^{i h t}$ for $i, h \in \mathbb{Z}$. Using that

$$
\text { p.v. } \frac{1}{2 \pi} \int_{-\pi}^{\pi} e^{i j t} \frac{d t}{\tan \frac{x-t}{2}}=\operatorname{sign}(j) e^{i j x}
$$

we find in this case $A_{b} f(x)=\operatorname{sign}(j l+h-l \gamma) e^{i(j k+j l+h-l \gamma) x}$. Hence for $b=\sum b_{j} e^{i j t}, f(t)=e^{i h t}$ we obtain

$$
A_{b} f(x)=\sum_{j} b_{j} \operatorname{sign}(j l+h-l \gamma) e^{i(j k+j l+h-l \gamma) x}
$$

To obtain the desired truncation we consider only frequencies of type $h=$ $-n(l+k), n \in \mathbb{N}$, and make the substitution $j=m+n$. Thus on $f(t)=\sum_{n} a_{n} e^{-i(l+k) n t}$, we find that

$$
A_{b} f(x) e^{i l \gamma x}=\sum_{n \in \mathbb{N}} \sum_{m \in \mathbb{Z}} a_{n} b_{m+n} \operatorname{sign}(l(m-n \beta-\gamma)) e^{i m(k+l) x}
$$

Finally we consider the projection on the closed subspace spanned by $e^{i m(k+l) x}$ for $m \in \mathbb{N}$. It follows that the matrix whose entries are $b_{m+n} \operatorname{sign}(m-n \beta-\gamma)$ acts boundedly on $l^{2}(\mathbb{N})$, with constant dominated by $C(\beta)\|b\|_{\infty}$, proving (2).

We now show that a Hankel operator $B$ is bounded if $\Pi(B)$ is. Indeed, if $\Pi(B)$ is bounded so is $\Pi(\bar{B})$, hence its adjoint $(\Pi(\bar{B}))^{*}$, and then so is $B$ because it differs from $\Pi(B)+(\Pi(\bar{B}))^{*}$ by a diagonal matrix with bounded entries $b_{2 n}$. 
For Toeplitz operators we argue in a similar way. As before, without loss of generality we may assume that $\beta=\frac{k}{l}$ with $k, l \in \mathbb{Z}, k \geq 1, k \neq l$, and $l \gamma \in \mathbb{Z}$. The auxiliary operator $A_{b}$ is defined now by

$$
\begin{aligned}
A_{b} f(x) & =\frac{1}{2 \pi} \text { p.v. } \int_{-\pi}^{\pi} b(-k x+l t) f(t) e^{-i t l \gamma} \frac{d t}{\tan \frac{x-t}{2}} \\
& \left.=\frac{1}{2 \pi} \text { p.v. } \int_{-\pi}^{\pi} b((l-k) x-l t) f(t-x)\right) e^{i \gamma l(t-x)} \frac{d t}{\tan \frac{t}{2}},
\end{aligned}
$$

and we use (3) with $\alpha=\frac{l}{k-l}$. We make act $A_{b}$ on $f(t)=e^{i(l-k) n t}, n \in \mathbb{N}$, obtaining now

$$
A_{b} f(x) e^{i l \gamma x}=\sum_{m \in \mathbb{Z}} b_{m-n} \operatorname{sign}(l(m-n \beta-\gamma)) e^{i m(l-k) x},
$$

and the proof is finished as before.

If $B=H_{b}$ is compact then $b$ can be assumed in $C(\mathbb{T})$. If $b_{n}$ are polynomials converging uniformly to $b$, the estimate above implies that $\Pi_{\beta, \gamma}(B)$ is the limit of the finite rank operators $\Pi_{\beta, \gamma}\left(H_{b_{n}}\right)$ and hence it is compact.

It is worth mentioning, though, that parts (a) and (b) are equivalent. This can be seen as follows. Let $P_{N}\left(x_{0}, \ldots, x_{N}, \ldots\right)=\left(x_{0}, \ldots, x_{N}, 0, \ldots\right)$ the standard coordinate projection, and let $J\left(x_{0}, \ldots, x_{N}\right)=\left(x_{N}, \ldots, x_{0}\right)$; note that $\sup _{N}\left\|P_{N} B P_{N}\right\|=\|B\|$ for every matrix $B$ and that for a $N \times N$ matrix $B, A=B J$ is Toeplitz (Hankel) if and only if $B$ is Hankel (Toeplitz). Also note that $\Pi_{\beta, \gamma}(B) J=\Pi_{\beta^{\prime}, \gamma^{\prime}}(A)$, with $\beta^{\prime}=-\beta$, $\gamma^{\prime}=\gamma-N \beta$. Assume that (a) holds, let $T$ be a bounded Toeplitz operator with symbol $\phi$, and let $T_{N}=P_{N} T P_{N}$. Then $T_{N} J$ is a Hankel matrix which equals $P_{N} \Gamma_{N} P_{N}$, where $\Gamma_{N}$ is the Hankel operator on $H^{2}$ having $\zeta^{N} \phi$ as a symbol. Hence, $\left\|\Pi_{\beta, \gamma}\left(\Gamma_{N}\right)\right\| \leq c(\beta)\left\|\zeta^{N} \phi\right\|_{\infty}=$ $c(\beta)\|\phi\|_{\infty}$ for every $\gamma$ and so

$$
\left\|P_{N} \Pi_{\beta, \gamma}\left(\Gamma_{N}\right) P_{N} J\right\|=\left\|P_{N} \Pi_{\beta, \gamma}\left(\Gamma_{N}\right) P_{N}\right\| \leq c(\beta)\|\phi\|_{\infty} .
$$

But $P_{N} \Pi_{\beta, \gamma}\left(\Gamma_{N}\right) P_{N} J=\Pi_{\beta, \gamma}\left(P_{N} \Gamma_{N} P_{N}\right) J=\Pi_{\beta, \gamma}\left(T_{N} J\right) J=\Pi_{\beta^{\prime}, \gamma^{\prime}}\left(T_{N}\right)$, with $\beta^{\prime}=-\beta, \gamma^{\prime}=\gamma-N \beta$. Since $\gamma$ is arbitrary, so is $\gamma^{\prime}$, and letting $N \rightarrow+\infty$ we conclude that $\left\|\Pi_{\beta^{\prime}, \gamma^{\prime}}(T)\right\| \leq c(\beta)\|\phi\|_{\infty}, \beta=-\beta^{\prime}$ for all $\gamma^{\prime}$, proving (b) (we thank the referee for this observation).

The following is an easy corollary of the theorem:

Corollary 3. Let $B$ be a compact Hankel operator. Then $B$ is the limit in the operator norm of its upper triangular truncations $B_{n}=$ $B-\Pi_{\beta, n}(B)$ if $\beta \neq-1$. 
We have seen that the theorem of Lacey-Thiele implies its periodic version and also Theorem 1 . We shall show that some converse is also true. We shall also show that the consideration of the operators $\Pi_{\beta, \gamma}$ gives some indication on the norm of the bilinear Hilbert transform.

The first point to note is that, conversely, Lacey-Thiele theorem follows from its periodic version. Indeed, in proving the estimate of Lacey-Thiele theorem, it is enough to consider $g, f$ supported in $[-\epsilon, \epsilon]$ (otherwise take $f(N x), g(N x))$. We want to bound

$$
\int_{-\infty}^{\infty}\left|\mathrm{p} . \mathrm{v} \cdot \int_{-\infty}^{\infty} g((k+l) x-l t) f(x-t) \frac{d t}{t}\right|^{r} d x
$$

The principal value is supported in $|x| \leq \frac{\epsilon(1+|l|)}{|k+l|}=\eta$. We call $F, G$ the periodized of $f, g$. We claim that for $|x| \leq \eta$ small enough,

p.v. $\int_{-\infty}^{\infty} g((k+l) x-l t) f(x-t) \frac{d t}{t}=$ p.v. $\int_{-\pi}^{\pi} G((k+l) x-l t) F(x-t) \frac{d t}{t}$.

Indeed, the last expression is nonzero only when there exists $t$ such that $(k+l) x-l t \in 2 m \pi+[-\epsilon, \epsilon], x-t \in 2 n \pi+[-\epsilon, \epsilon]$ for some integers $m, n$, which is equivalent to $x \in 2 \pi(m-l n) /(k+l)+[-\eta, \eta]$. If $\eta \leq \frac{\pi}{2|k+l|}$, only $m=n=0$ contribute, establishing the claim. Now, changing $1 / t$ into $1 /\left(2 \tan \frac{t}{2}\right)$ as before shows that Lacey-Thiele theorem follows from (3).

Assume we know that $\Pi$ is a bounded operator when restricted to Hankel operators. Clearly $\Pi_{1,-1}$ is also a bounded operator when restricted to Hankel operators. We claim that we have an a priori estimate for the periodic bilinear Hilbert transform $H(b, f)$ with $b$ and $f$ trigonometric polynomials. More precisely, we will prove that

$$
\|H(b, f)\|_{2} \leq C\|b\|_{\infty}\|f\|_{2},
$$

with a constant $C$ which only depends on the norm of $\Pi$. Indeed,from the above computation, it follows that, whenever $f(t)=\sum_{n} a_{n} e^{-2 i n t}$,

$$
H(b, f)(x)=\sum_{n \in \mathbb{N}} \sum_{m \in \mathbb{Z}} a_{n} b_{m+n} \operatorname{sign}(m-n) e^{2 i m x} .
$$

So $H(b, f)(x)=-b(2 x) f(x)+\Pi\left(H_{b}\right) f(x)$. When $f(t)=\sum_{n} a_{n} e^{-i(2 n+1) t}$, this formula has to be changed into $H(b, f)(x)=-b(2 x) f(x) e^{2 i x}+$ $\Pi_{1,-1}\left(H_{b}\right) f(x)$. We find the required inequality for $\|H(b, f)\|_{2}$ when $f$ is a Taylor polynomial, cutting it into even and odd frequencies. 
We conclude for $f$ a trigonometric polynomial taking real and imaginary parts. So the boundedness of $\Pi$ implies the existence of the constant $C\left(-\frac{1}{2}, 2, \infty\right)$, or, by duality (as shown in $[\mathbf{L T 3}]$ ), the existence of the constant $C(1,2,2)$, the original Calderón conjecture.

Finally, for some values of $p, q$, we give some information on the behavior of the constant $C(\alpha, p, q)$ when $\alpha$ tends to $0,-1$, or $\infty$. If we consider Toeplitz operators and take $l=k-1$, we see that $C(k, 2, \infty)$ cannot remain bounded when $k$ tends to $+\infty$, otherwise $\Pi\left(T_{b}\right)$ would be bounded for $T_{b}$ bounded. The same is valid for $k$ tending to $-\infty$. By duality, we find that $C(\alpha, 2,2)$ cannot remain bounded when $\alpha$ tends to -1 . These properties could have been directly obtained from the consideration of the bilinear Hilbert transform. Using Hankel operators we find a bound below for these constants.

Lemma 4. $C( \pm N, 2, \infty) \geq c \ln N$.

Let us take $k=-l+1$, so that $\beta=-1+\frac{1}{l}$ and $\alpha=-l$. Then it is possible to find $\gamma$ so that $\Pi_{-1, k}=\Pi_{\beta, \gamma}$. Using the fact that the operator giving the partial sum of order $N$ of the Fourier series of a function in $B M O$ has norm equivalent to $\ln N$, we get that the norm of $\Pi_{-1, k}$ is equivalent to $\ln k$. Since it is bounded by $C(\alpha, 2, \infty)$, we get the estimate of the lemma for negative integers. The choice $k=-l-1$ gives the other case.

We also point out that in an analogous way, the Lacey-Thiele theorem can be used to obtain a version of our theorem for "continuous" Hankel operators, i.e. the bounded integral operators defined in $L^{2}(0,+\infty)$ of type

$$
H_{b} f(x)=\int_{0}^{\infty} b(x+y) f(y) d y .
$$

For instance, if $H_{b}$ is bounded in $L^{2}(0, \infty)$, so is

$$
\Pi\left(H_{b}\right) f(x)=\int_{0}^{\infty} b(x+y) \operatorname{sign}(x-y) f(y) d y
$$

We finish this section by posing two open problems suggested by the proof.

The first concerns finite $N \times N$ Hankel or Toeplitz matrices; for fixed $\beta, \gamma$, let $c_{N}$ denote the norm of the truncation operator $\Pi_{\beta, \gamma}$ when restricted to $N \times N$ Hankel matrices. Which is the behaviour of $c_{N}$ as $N \rightarrow \infty$ ? Note that our result does not apply to such Hankel matrices. 
The second concerns weighted Cauchy integral operators: let $b(\zeta, z)$ bounded and consider

$$
T_{b} f(z)=\int_{\mathbb{T}} \frac{b(\zeta, z) f(\zeta)}{1-\bar{\zeta} z} d \zeta
$$

For which symbols $b$ is $T_{b}$ bounded in $H^{2}(\mathbb{D})$ ? (in our proof we used symbols of type $\left.b\left(\zeta^{k} z^{l}\right), b\left(\zeta^{k} \bar{z}^{l}\right)\right)$.

\section{Hankel operators in the trace class}

We shall first prove that the Schatten classes $S_{p}$ are globally preserved by the truncation operators $\Pi_{\beta, \gamma}$ when $1<p<\infty$, as said in the introduction. Our proof follows from well known arguments. We give it for completeness.

Remember that, for $p>0$, given a compact operator $T$ on a Hilbert space, we say that $T$ belongs to the Schatten class $S_{p}$ if $\sum_{j} s_{j}^{p}<\infty$, where

$$
s_{j}=\{\inf \|T-E\|: \operatorname{rank} E \leq j\} .
$$

We will make use of two facts. The first one is that the Schatten classes are closed ideals in the algebra of bounded operators (see [GK1]). The second one is that the Hilbert transform maps also continuously $L^{p}$ into itself, for $1<p<\infty$, when extended to $S_{p}$-valued functions (see $[\mathbf{R u}]$ ). For $t \in \mathbb{T}$, let us define $R_{t}$ as the diagonal matrix with diagonal entries given by $e^{i n t}, n=0,1,2, \ldots$. Then an elementary computation shows that, when $B=\left(b_{m, n}\right)_{m, n \geq 0}$, the matrix $R_{s} B R_{t}$ has entries $b_{m, n} e^{i(m s+n t)}$. Let us now go back to the truncation operators $\Pi_{\beta, \gamma}$, for which we shall prove that they are uniformly bounded in the Schatten classes $S_{p}$. Without loss of generality we may assume as before that $\beta=\frac{k}{l}$ with $k, l \in \mathbb{Z}, k \geq 1$, and $l \gamma \in \mathbb{Z}$. For $B \in S_{p}$, let us consider the $S_{p}$-valued function

$$
B(t)=e^{-i \gamma t} R_{l t} B R_{-k t}
$$

whose entries are given by $b_{m, n} e^{i(m l-k n-\gamma) t}$. Then the Cauchy projection of $B(t)$, extended to $S_{p}$-valued functions, is equal to

$$
C B(t)=e^{-i \gamma t} R_{l t} \Pi_{\beta, \gamma}(B) R_{-k t} .
$$

We know that this extension of the Cauchy projection is uniformly bounded in $L^{p}$. Moreover, it is easy to see that the norm in $S_{p}$ of $B(t)$ does not depend of $t$, and is equal to $\|B\|_{S_{p}}$. The same is valid for $C B(t)$, for which we find $\left\|\Pi_{\beta, \gamma}(B)\right\|_{S_{p}}$. The conclusion follows at once.

Let us now consider trace class operators, that is the case $p=1$. 
Theorem 5. The operator $\Pi$ is not bounded in $S_{1}$, even when restricted to Hankel operators.

Proof: Let us consider the Hankel operators $H_{r}$ with symbol $b_{r}(z)=$ $\frac{1-r^{2}}{1-r z}$. The definition shows that $H_{r} f(z)=f(r) b_{r}(z)$, that is, $H_{r}$ is the projection on the subspace generated by the function $b_{r}$. In particular $H_{r}$ is of rank 1 , and of norm 1 . Since $b_{k}=\left(1-r^{2}\right) r^{k}$,

$$
\begin{aligned}
\Pi\left(H_{r}\right) f(z) & =\left(1-r^{2}\right) \sum_{m}\left(\sum_{n \leq m} a_{n} r^{n+m}\right) z^{m} \\
& =\left(1-r^{2}\right) \sum_{n} a_{n} r^{n} \sum_{m \geq n} r^{m} z^{m} \\
& =\frac{1-r^{2}}{1-r z} \sum_{n} a_{n} r^{2 n} z^{n}=b_{r}(z) f\left(r^{2} z\right) .
\end{aligned}
$$

We set, for $0<r<1$,

$$
U_{r} f(z)=\frac{1-r^{2}}{1-r z} f(r z)
$$

Since the quotient $\left|b_{r^{2}} / b_{r}\right|$ is bounded above and below and $S_{1}$ is an ideal, the norms in the trace class of $\Pi\left(H_{r}\right)$ and $U_{r^{2}}$ are equivalent. So the unboundedness of $\Pi$ for Hankel operators in the trace class follows from the following lemma.

Lemma 6. The norm of $U_{r}$ in the trace class $S_{1}$ is equivalent to $\ln \left(\frac{1}{1-r}\right)$.

Proof: For the lower bound we adapt a method which is employed to find necessary conditions on symbols of Hankel operators. $L^{2}(d V)$ denotes the ordinary Lebesgue space on the unit disc $\mathbb{D}$ endowed with Lebesgue measure $d V$. We shall use the fact that for $A$ a bounded operator from $H^{2}$ into $L^{2}(d V)$, since $S_{1}$ is an ideal, the norm in the trace class of the operator $A U_{r} A^{*}$ is bounded by $\|A\|^{2}\left\|U_{r}\right\|_{S_{1}}$. We combine this with the fact that the trace class norm is given by

$$
\|B\|_{S_{1}}=\sup \sum_{i}\left|\left\langle B e_{i}, e_{i}\right\rangle\right|
$$


the supremum being taken over all orthonormal systems. Hence, if $\left(e_{k}\right)_{k=1}^{K}$ is an orthonormal system in $L^{2}(d V)$, then

$$
\sum_{k=1}^{K}\left|\left\langle U_{r} A^{*} e_{k}, A^{*} e_{k}\right\rangle\right| \leq\|A\|^{2}\left\|U_{r}\right\|_{S_{1}}
$$

For $k=1,2, \ldots, K$, let us set $w_{k}=r e^{i \theta_{k}}$, with $\theta_{k}=k(1-r)$, and $K$ the biggest integer such that $K(1-r) \leq \pi / 4$. It is clear that $K \simeq(1-r)^{-1}$. Now, let $A$ be the map given by

$$
A f=(1-r)^{-1 / 2} \sum_{k=1}^{K} f\left(w_{k}\right) \chi_{D_{k}}
$$

where $\chi_{D_{k}}$ denotes the characteristic function of the disc $D_{k}$ which has center $w_{k}$ and radius $\eta(1-r)$. For $0<\eta<1 / 2$ fixed, small enough, the discs $D_{k}$ are disjoint. It follows from this fact and from the mean value inequality that

$$
\|A f\|_{L^{2}(d V)}^{2} \leq C_{\eta}(1-r)^{-1} \int_{r-\eta(1-r)<|z|<r+\eta(1-r)}|f(z)|^{2} d V(z) \leq C_{\eta}\|f\|_{H^{2}}^{2} .
$$

So $A$ is a bounded operator from $H^{2}$ into $L^{2}(d V)$, with norm bounded independently of $r$. We set $e_{k}=c_{\eta}(1-r)^{-1} \chi_{D_{k}}$. For $c_{\eta}$ well choosen, $\left(e_{k}\right)_{k=1}^{K}$ is an orthonormal system in $L^{2}(d V)$. Moreover $A^{*}$ maps $e_{k}$ to $f_{k}(z)=\frac{\left(1-r^{2}\right)^{1 / 2}}{1-\overline{w_{k}} z}$. It follows from this choice that there is some constant $C$, which does not depend on $r$, such that

$$
\sum_{k=1}^{K} \frac{1}{2 \pi}\left|\int_{-\pi}^{+\pi} \frac{1-r^{2}}{1-r e^{i t}} \times \frac{\left(1-r^{2}\right)^{1 / 2}}{1-\overline{w_{k}} r e^{i t}} \times \frac{\left(1-r^{2}\right)^{1 / 2}}{1-w_{k} r e^{-i t}} d t\right| \leq C\left\|U_{r}\right\|_{S_{1}}
$$

We now use the reproducing property of the Szegö kernel to replace this last inequality by

$$
\sum_{k=1}^{K} \frac{1-r^{2}}{\left|1-r w_{k}\right|} \leq C\left\|U_{r}\right\|_{S_{1}}
$$

We observe now that $\left|1-r w_{k}\right| \simeq\left(1-r^{2}\right) k$. This proves the bound from below. 
Now we will prove the upper bound. Let us denote by $M_{r}$ the operator of multiplication by the function $\varphi(z)=\left(\frac{1-r^{2}}{1-r z}\right)^{1 / 2}$, and $P_{r}$ the operator defined on $H^{2}$ by $P_{r} f(z)=f(r z)$. We claim that $U_{r}$ and $M_{r} P_{r} M_{r}$ have equivalent norms in the trace class. Indeed,

$$
U_{r} f(z)=M_{r} P_{r} M_{r} f(z) \frac{\left(1-r^{2} z\right)^{1 / 2}}{(1-r z)^{1 / 2}}
$$

and multiplication by a function which is bounded below and above preserves the norm, up to a constant. Let us consider the operator $V_{r}$, defined in the whole $L^{2}(\mathbb{T})$ by the same expression $M_{r} P_{r} M_{r}$, where $M_{r}$ denotes again multiplication by the same function $\varphi$, restricted to the unit circle, and now $P_{r}$ denotes convolution with the Poisson kernel. Clearly the operator $V_{r}$ is an extension of $U_{r}$ and so it is sufficient to prove that

$$
\left\|V_{r}\right\|_{S_{1}} \leq C \ln \left(\frac{1}{1-r}\right)
$$

as an operator in $L^{2}(\mathbb{T})$. Now the adjoint of $M_{r}$ as an operator on $L^{2}(\mathbb{T})$ is multiplication by $\left(\frac{1-r^{2}}{1-r e^{-i t}}\right)^{1 / 2}$, which has the same modulus as $\varphi\left(e^{i t}\right)$. It follows that $V_{r}$ and $M_{r}^{*} P_{r} M_{r}$ have same norm. This last operator is self adjoint and positive since $P_{r}$ is. Now, recall that for positive operators the trace class norm is of course given by the trace, and this can be computed in any orthormal basis (this follows from (4) too). Hence,

$$
\left\|V_{r}\right\|_{S_{1}}=\left\|M_{r}^{*} P_{r} M_{r}\right\|_{S_{1}}=\sum\left\langle M_{r}^{*} P_{r} M_{r} f_{k}, f_{k}\right\rangle
$$

where $f_{k}(t)=e^{-i k t}$. Now, $M_{r} f_{k}(t)=\varphi(t) e^{-i k t}=\sum_{l} \hat{\varphi}(l) e^{i(l-k) t}$, $P_{r} M_{r} f_{k}(t)=\sum_{l} \hat{\varphi}(l) r^{|l|} e^{i(l-k) t}$ and therefore by Plancherel formula,

$$
\left\|V_{r}\right\|_{S_{1}}=\sum_{k} \sum_{l} r^{|k-l|}|\hat{\varphi}(l)|^{2} .
$$

Summing first in $k$, and using Plancherel formula again, we get

$$
\left\|V_{r}\right\|_{S_{1}}=(1-r)^{-1} \frac{1}{2 \pi} \int_{-\pi}^{+\pi} \frac{1-r^{2}}{\left|1-r e^{i t}\right|} d t \simeq \ln \left(\frac{1}{1-r}\right) .
$$

This finishes the proof of the lemma and of Theorem 5 . 
Let us recall that the operator $H_{b}$ is in the Schatten class $S_{1}$ if and only if the symbol $b$ belongs to the Besov space $B^{1}$, where $B^{1}$ is the space of holomorphic functions $b$ in the unit disc such that $\int_{\mathbb{D}}\left|b^{\prime \prime}(z)\right| d V(z)<\infty$. We give now a sufficient condition for $\Pi\left(H_{b}\right)$ to be in $S_{1}$ which is of the same type, but stronger.

Theorem 7. Assume that the function b satisfies the condition

$$
\int_{\mathbb{D}}\left|b^{\prime \prime}(z)\right| \ln \left(\frac{1}{(1-|z|)}\right) d V(z)<\infty
$$

Then the truncated Hankel operator $\Pi\left(H_{b}\right)$ is in the trace class.

Proof: The proof is straightforward. We use the fact that the holomorphic function $b$ may be written as

$$
b(z)=\frac{1}{\pi} \int_{\mathbb{T}} N^{2} b(\zeta) \frac{\left(1-|\zeta|^{2}\right)}{(1-z \bar{\zeta})} d V(\zeta)
$$

with $N^{2} b$ the second derivative given by $N^{2} b(z)=\left(z \frac{\partial}{\partial z}+I\right)\left(z \frac{\partial}{\partial z}+\right.$ $2 I) b(z)$. It follows that

$$
H_{b}=\int_{\mathbb{T}} N^{2} b(\zeta) H_{\zeta} d V(\zeta)
$$

where $H_{\zeta}$ is the Hankel operator related to the function $b_{\zeta}(z)=\frac{1-|\zeta|^{2}}{1-\bar{\zeta} z}$. Moreover,

$$
\Pi\left(H_{b}\right)=\int_{\mathbb{T}} N^{2} b(\zeta) \Pi\left(H_{\zeta}\right) d V(\zeta)
$$

The integral is well defined since the hypothesis on $b$ implies

$$
\int_{\mathbb{D}}\left|N^{2} b(\zeta)\right| \ln \left(\frac{1}{1-|\zeta|}\right) d V(\zeta)<\infty
$$

and, by the lemma, $\left\|\Pi\left(H_{\zeta}\right)\right\|_{S_{1}} \simeq \ln \left(\frac{1}{1-|z|}\right)$, proving that $\Pi\left(H_{b}\right)$ is in the trace class. 
We will finish showing that the sufficient condition given in the last statement is not necessary. More precisely,

Theorem 8. Let $\rho$ be a positive increasing function on $[0,1)$ with limit $\infty$ at 1 . Then there exists a symbol b such that $\Pi\left(H_{b}\right)$ is in the trace class while

$$
\int_{\mathbb{D}}\left|b^{\prime \prime}(z)\right| \rho(|z|) d V(z)=+\infty .
$$

Proof: For $b(z)=\sum_{n>1} a_{n} z^{3^{n}}, \Pi\left(H_{b}\right)$ is clearly in the trace class if the sum of the absolute values of the entries of its matrix is bounded, that is $\sum_{n} 3^{n}\left|a_{n}\right|<\infty$. It is easy to find sequences $\left(a_{n}\right)$ for which this quantity is finite but, for a given $\rho$, the integral above is not.

\section{References}

[ACN] J. Angelos, C. Cowen And S. NARAyan, Triangular truncation and finding the norm of a Hadamard multiplier, Linear Algebra Appl. 170 (1992), 117-135.

[GK1] I. C. GohberG AND M. G. Krein, "Introduction to the theory of linear nonselfadjoint operators," Translations of Mathematical Monographs 18, Providence, R.I. AMS, 1969.

[GK2] I. C. GohBerg AND M. G. KREIN, "Theory of Volterra operators and its applications," Translations of Mathematical Monographs 24, Providence, R.I. AMS, 1970.

[DS] N. Dunford AND J. Schwartz, "Linear operators II," Interscience Publishers, Wiley \& Sons, New-York, 1963.

[KP] S. KWAPIEn And A. Pelczynski, The main triangle projection in matrix spaces and applications, Studia Math. 34 (1970), 43-68.

[LT1] M. LACEY AND C. ThiELE, $L^{p}$ estimates for the bilinear Hilbert transform, Proc. Nat. Acad. Sci. U.S.A. 94(1) (1997), 33-35.

[LT2] M. LACEy AND C. ThiELE, $L^{p}$ estimates for the bilinear Hilbert transform for $2<p<\infty$, Ann. of Math. 146(3) (1997), 693-724.

[LT3] M. LACEY AND C. ThiELE, On Calderón's conjecture for the bilinear Hilbert transform, Proc. Nat. Acad. Sci. U.S.A. 95(9) (1998), 4828-4830.

[Pe] V. V. PELler, Hankel operators of class $\mathcal{C}_{p}$ and their application (rational approximation, Gaussian processes, the problem of majorizing operators), Russian Acad. Sci. Sb. Math. 41 (1982), $443-479$. 
[Po] S. C. Power, "Hankel operators on Hilbert space," Research Notes in Mathematics 64, Pitman, London, 1982.

[Ru] J. L. RuBio DE FRANCIA, Martingales and integral transforms of Banach space valued functions, in "Probability and Banach spaces," (J. Bastero \& M. San Miguel, eds.), Lecture Notes in Math. 1221, Springer Verlag, Berlin-New York, 1986, pp. 195-222.

\author{
Aline Bonami: \\ Université d'Orléans \\ B.P. 6759 \\ 45067 Orléans Cedex 2 \\ FRANCE \\ e-mail: bonami@labomath.univ-orleans.fr
}

\author{
Joaquim Bruna: \\ Departament de Matemàtiques \\ Universitat Autònoma de Barcelona \\ 08193 Bellaterra (Barcelona) \\ SPAIN \\ e-mail: bruna@mat.uab.es
}

Primera versió rebuda el 2 de juny de 1998, darrera versió rebuda el 8 de març de 1999 